\title{
Fragmentation of cosmic-string loops
}

\author{
Thomas York \\ NASA/Fermilab Astrophysics Center * \\ Fermi National Accelerator Laboratory, P.O.B. 500 \\ Batavia, IL 60510
}

January 25, 1989

\begin{abstract}
The fragmentation of cosmic string loops is discussed, and the results of a simulation of this process are presented. The simulation can evolve any of a large class of loops essentially exactly, including allowing fragments that collide to join together. Such reconnection enhances the production of small fragments, but not drastically. With or without reconnections, the fragmentation process produces a collection of non-self-intersecting loops whose typical length is on the order of the persistence length of the initial loop.
\end{abstract}

\section{Introduction}

It has been suggested that string-like topological defects produced in a phase transition near the grand unification scale could have interesting cosmological consequences, and in particular that these "cosmic strings" might be the density inhomogeneities responsible for initiating the formation of such structures as galaxies and clusters of galaxies. (For reviews see Turok[1], and Vilenkin[2].) This scenario relies on the idea that the evolution of the string network eventually settles down to a "scaling solution" in which, at any time, there is a network of long strings plus many short loops, with the persistence length of the long string, the typical spacing between strings, and the typical loop size all being proportional to

Permanent addreas: Cornell University Department of Physics, Clark Hall, Ithaca, NY 14853 


\section{ORIGINAL PAGE IS}

OF POOR QUALITY

the time $t$. In this case the energy density in string scales like $t^{-2}$ and remains a fixed fraction of the total energy density. To get such behavior it is necessary for strings to be able to reconnect the other way, or "intercommute", when they intersect each other, an assumption now supported by several calculations $[3,4]$. Long strings can then intersect themselves and chop off closed loops, thus losing energy as they must for scaling to occur. The closed loops may also self-intersect and further break up (which is the topic of this paper), but if not they can at least decay by gravitational radiation, each loop losing energy at a rate $\dot{\mathrm{E}}=-\Gamma \mathrm{G} \mu^{2}$ where $\mu$ is the string tension and $\Gamma$ is a factor which depends on the loop's shape but not on its length $[5,6,7,8]$. (Here and throughout this article $c=1$.) It will also occur that loops will collide with and join onto long strings or other loops, so these processes need to be understood too.

The simulations of Albrecht and Turok[9,10], and of Bennett and Bouchet [11], which numerically integrate the string equations of motion in an expanding universe, taken together with analytical modelling $[12,13,14]$, now strongly suggest that string evolution will indeed approach a scaling solution from a wide range of initial conditions. These two approaches are to some degree complementary: numerical simulations are necessary to check the assumptions and fix the parameters of analytical models, which in turn are necessary to understand the long time scale behavior of the network (such as the accumulation of short loops and their slow decay by gravitational radiation) since these simulations can study times only up to a few times the initial time before effects due to the finite size of the box in which the simulation is done become significant. Since these simulations discretize the string equations of motion they are also limited in the smallest scale phenomena that can be resolved. One approach to understanding possible resolution effects would be to vary the resolution and see what else changes. Another approach, taken here, is to study the simpler problem of a single loop in flat space-time, in which case discretization can be avoided.

I have studied loop fragmentation in flat space-time by means of a simulation which, for a large class of initial conditions, evolves the string essentially exactly between crossings, describes the string after an intercommutation as precisely as before, and allows the production of arbitrarily small loops. It is known that there are many loop solutions which do not self-intersect $[15,16,17]$, so it is natural to ask whether fragmentation eventually produces a collection of loops which are all non-self-intersecting; and, if it does, to study the properties of these stable 


\section{ORIGINAL PAGE IS}

OF POOR QUALITY

fragments. Scherrer and Press also have studied loop fragmentation by choosing loops randomly from a certain class and explicitly evolving them, and find that a finite number of non-self-intersecting fragments are produced [18]. The principle differences between the present work and theirs are that I sample the space of loops in a different way, more naturally related to the loops produced off long string; and that $I$ include the joining together of fragments that collide. This reconnection may be important to understand since each intercommutation creates 4 kinks and so the joining of two loops is not the inverse of one breaking in two. Since the statistical mechanics of string strongly favors short loops $[19,20]$, it is reasonable to expect that the joining of fragments that collide and the associated creation of kinks will, by helping the string explore its phase space, lead in the end to smaller fragments. We will see that this is indeed what happens.

The following section presents the equations of motion of a loop and describes the simulation. Section 3 presents results on the length and velocity distributions of the fragments and section 4 discusses the implications of these results for our understanding of the evolution of the whole string network.

\section{Loop Motion and the Simulation}

For loops whose size is small compared to $t$ and large compared to the string width, the equations of motion may be taken to be:[21]

$$
\begin{gathered}
\ddot{\mathbf{x}}(\sigma, t)=\mathbf{x}^{\prime \prime}(\sigma, t) \\
\dot{\mathbf{x}} \cdot \mathbf{x}^{\prime}=0
\end{gathered}
$$

and

$$
(\dot{\mathbf{x}})^{2}+\left(\mathbf{x}^{\prime}\right)^{2}=1
$$

where $\dot{\mathbf{x}} \equiv \partial \mathbf{x} / \partial t$ and $\mathbf{x}^{\prime} \equiv \partial \mathbf{x} / \partial \sigma$, and $\sigma$ is a parameter which varies along the string in such a way that the energy per unit $\sigma$ is equal to a constant, $\mu$, the string tension. The general solution to Eq. (1) is

$$
\mathbf{x}(\sigma, t)=\frac{1}{2}\left[\mathbf{a}\left(\sigma_{-}\right)+\mathbf{b}\left(\sigma_{+}\right)\right]
$$

where $\sigma_{ \pm} \equiv \sigma \pm t$, and

$$
\left(\mathbf{a}^{\prime}\right)^{2}=\left(\mathbf{b}^{\prime}\right)^{2}=1
$$




\section{ORIGINAL PAGE IS \\ OF POOR QUALTYY}

in order to satisfy equations (2) and (3). Let $L$ be the total length of $\sigma$ around the loop. Then, in order for the loop to be closed, $\mathbf{x}(\sigma, t)=\mathbf{x}(\sigma+L, t)$ or

$$
-\left[\mathbf{a}\left(\sigma_{-}+L\right)-\mathbf{a}\left(\sigma_{-}\right)\right]=\left[\mathbf{b}\left(\sigma_{+}+L\right)-\mathbf{b}\left(\sigma_{+}\right)\right]=\mathbf{\Delta}
$$

where $\Delta$ is a constant. Note that $\mathbf{x}(\sigma, t+L)=\mathbf{x}(\sigma, t)+\Delta$. After a time $\delta t=L$ the loop returns to its original shape but translated by $\Delta$, so the center of mass velocity is $\mathbf{v}=\Delta / L$. In the center of mass frame $\mathbf{v}=0$ and, by Eq. (6), the functions $a$ and $b$ are both periodic. One may then think of $a$ and $b$ as closed curves, parametrized by their length in order to satisfy Eq. (5). A natural way to sample the space of such curves is to consider closed random walks.

I consider the class of loops for which a and $\mathbf{b}$ are closed random walks of $\mathrm{N}$ equal sized straight steps. Equivalently $\mathbf{a}^{\prime}$ and $\mathbf{b}^{\prime}$ each take on $\mathbf{N}$ discrete values $\mathbf{a}_{i}^{\prime}$ and $\mathbf{b}_{i}^{\prime}$ lying on the unit sphere, with

$$
\sum_{i=1}^{N} \mathbf{a}_{i}^{\prime}=\sum_{i=1}^{N} \mathbf{b}_{i}^{\prime}=0
$$

The curves $\mathbf{a}, \mathbf{b}$ and $\mathbf{x}(\sigma, t)=\frac{1}{2}\left[\mathbf{a}\left(\sigma_{-}\right)+\mathbf{b}\left(\sigma_{+}\right)\right]$all have a persistence length $\ell_{p}=L / N$, (aside from the correlation among the $\mathbf{a}_{i}^{\prime}$ and $\mathbf{b}_{i}^{\prime}$ due to the constraint expressed in Eq. (7), which should be only a small effect for large $N$ ). This provides a natural way of relating these loops to the full string network, which is also described in terms of a persistence length.

: The first task for the simulation is to generate random loops of this type, i.e. to generate $N$ unit vectors $a_{i}^{\prime}$ which sum to zero, but are otherwise random, and similarly for the $\mathbf{b}_{i}^{\prime}$. The code first chooses all the $\mathbf{a}_{i}^{\prime}$ to be along the $x, y$ and $z$ axes, making sure to go in the $+x$ and $-x$ directions an equal number of times, (and likewise for $y$ and $z$,) to ensure that $a$ is closed. Next a pair of these vectors is selected and they are rotated by a random angle about the axis defined by their mean. This operation preserves $\sum \mathbf{a}_{i}^{\prime}$, so a remains closed. This operation is repeated many times, selecting a different pair each time, in order to randomize the $a_{i}^{\prime}$. An independent set of $b_{i}^{\prime}$ is similarly constructed.

After defining an initial 'parent' loop in this manner, the simulation searches for crossings in successive time intervals until it finds one. The intercommutation probability is taken to be 1 , so this first crossing results in the loop breaking in two; subsequent crossings may be between distinct loops in which case they join together, or 'reconnect'. The search for crossings then resumes, starting at the time 
of the intercommutation. The motion of a (non-self-intersecting) loop is a periodic oscillation plus a constant velocity translation, so when all loops have survived for one period we know that none are self-intersecting. This is the criterion I use for the end of the fragmentation process. It is possible for distinct fragments to collide after this time, but this was found to be a rare occurence. Figure (1) shows a typical loop with $N=10$, at a time shortly before the first crossing, and at a later time after fragmentation is complete. In order to save on computation time, fragments shorter than a certain length $\ell_{c}$ are checked only for self-intersections and not for collisions with other strings. The effect of this cutoff is discussed in the following section.

The simulation must find where the string crosses itself, i.e. find $t$ and distinct $\sigma_{1}$ and $\sigma_{2}$ for which $\mathbf{x}\left(\sigma_{1}, t\right)=\mathbf{x}\left(\sigma_{2}, t\right)$. Loops of the type considered here initially have $\mathrm{N}$ evenly spaced kinks propagating in each direction (left and right) around the loop, joined by straight segments. When a left-moving kink and a right-moving kink pass each other, a straight segment appears between them and grows longer as they draw apart. Eventually a right-moving kink coming in from the left and a left-moving kink coming in from the right shrink the segment back down until it disappears. The simulation searches for crossings in a given time interval by finding all straight sections whose lifetimes overlap that interval, and checking each pair of them. On each straight segment, $\mathbf{x}^{\prime}$ and $\dot{\mathbf{x}}$ are constant. The world sheet of such a segment is a parallelogram, a subset of the world-sheet of an infinite moving line which may be parametrized as $\mathbf{x}(\sigma, t)=\mathbf{x}_{0}+\mathbf{x}^{\prime} \sigma+\dot{\mathbf{x}} t$ for a suitable choice of $x_{0}$. The time $t$ of crossing of two such lines, and the $\sigma_{1}$ and $\sigma_{2}$ where they cross, may easily be found in closed form; then if $\left(\sigma_{1}, t\right)$ and $\left(\sigma_{2}, t\right)$ lie in the regions of $\sigma, t$ space corresponding to their respective straight segments, a crossing has been found. In this way all pairs are checked and the earliest crossing in the time interval is found.

Next the strings at the crossing point must be intercommuted, breaking one loop into two (if a loop has self-intersected), or joining two into one (if one loop has intersected another). Afterwards the string will still consist of straight segments and kinks. This means that each fragment may still be described by an and a b which consist of straight sections, but now the lengths of these straight sections may be unequal, and since the fragment may have center of mass motion, $a$ and b will in general not be closed. The simulation, taking this properly into account, either defines two new a (b) functions using the a (b) of the loop which has self- 
intersected, or defines a new a (b) using the a's (b's) of the two loops which have intersected, as the case may be. The description of the string is just as precise after intercommutation as before.

A peculiarity of the class of loops considered here is that fragments with less than 5 kinks are not produced. These loops will consist of straight segments and kinks in any frame, so 4 kinks ( 2 moving left, 2 right) is the minimum number since Eq. (7) must hold in the rest frame. Such a 4 kink loop is highly degenerate: in its rest frame its motion lies in a plane. Only a set of zero measure of initial loops would allow the production of such loops. When a loop breaks in two, each fragment gets 2 of the 4 new kinks (1 left-, 1 right-moving), so if a 5 kink fragment is created it will have 3 old kinks, of which 2 will be going the same way. This implies that the shortest fragment that an initial loop can break off is $\ell_{p}=L / N$, the spacing between left-(right-)moving kinks.

\section{Results}

To find the probability, $\mathrm{P}_{0}$, that loops of the type described above do not selfintersect, a large number of loops (e.g. $10^{4}$ for $N=9$ ) were checked for selfintersections. Figure (2) shows the results. The curve plotted is $P_{0}=13\left(2.45^{-N}\right)$. Before $N$ has become very large, $P_{0}$ is already tiny; in a stochastic fragmentation process characterized by a fixed probability of fragmentation $q$, values of $q$ so close toiunity would imply that a loop would almost al ways break into an infinite number of fragments $[22,18,13,14]$, but this is not observed. Indeed, if reconnections are neglected, the fact that (for this class of loops) every fragment has at least 5 kinks whereas each intercommutation produces only 4 means that in breaking a loop up one eventually runs out of kinks. If $N_{f}$ fragments are produced (by $N_{f}-1$ selfintersections) the number of kinks will be $2 N$ from the parent loop plus $4\left(N_{f}-1\right)$ from intercommutations, and must be at least $5 N_{f}$, so $N_{f} \leq 2 N-4$. When reconnections are included this limit does not hold.

The length distribution of the fragments remaining when fragmentation is complete was measured for various values of $N=L / \ell_{p}$, and for comparison the reconnection of fragments that collide was included in some runs and neglected in others. Let $n(x) d x$ be the mean number of fragments per parent loop produced with $x \equiv \ell / \ell_{p}$ lying between $x$ and $x+d x$. Then the fraction of the parent loop's energy which (on the average) goes into fragments in this size range is 
$N^{-1} x \mathrm{n}(x) d x \equiv \epsilon(x) d x$. For small $N$ (5 was the smallest $N$ I considered), $\epsilon(x)$ is peaked around $x=1.4$, and the production of short loops is slightly enhanced by including reconnections. As $N$ is increased, $\epsilon(x)$ at small $x$ increases. This increase is only slight if reconnections are neglected, but if they are included it is more pronounced: as $\mathrm{N}$ is increased the left side of the peak fills in until $\epsilon(x)$ is roughly flat for small $x$. Figure (3), showing $\epsilon(x)$ for $N=15$ with and without reconnections, illustrates this behavior. At larger $x, \epsilon(x)$ falls like $x^{-2}$. The number of parent loops evolved to produce these plots was 90 with reconnections, and 400 without. As mentioned above the simulation does not check loops shorter than a certain length $\ell_{c}$ for reconnection, although it does find their self-intersections and allow them to break up. Neglecting reconnection is therefore equivalent to setting $\ell_{c}=L / 2$. (There must be at least 2 fragments longer than $\ell_{c}$ to find a reconnection.)

An interesting quantitative measure of the typical length of fragments produced is

$$
\alpha=\frac{\int \epsilon(x) x^{\frac{1}{2}} d x}{\int \epsilon(x) d x}
$$

This is interesting because if it is assumed that all the loops produced at time $t$ in the scaling solution have the same length $\ell=\gamma t$ then, in the radiation dominated era, the energy densities in loops $\left(\rho_{l}\right)$, in long string $\left(\rho_{s}\right)$ and in gravitational radiation from string $\left(\rho_{g r}\right)$ are related by $[12,23,10] \rho_{l} / \rho_{0} \propto \rho_{g r} / \rho_{c} \propto \gamma^{\frac{1}{2}}$. Since in the scaling solution the persistence length is proportional to $t$ we may say the loops have length $\ell=x \ell_{p}$ and that these density ratios are proportional to $x^{\frac{1}{2}}$. If, as is actually the case, the loops have a range of sizes, we need to take a mean, weighting each value of $x^{\frac{1}{2}}$ by the amount of energy going into loops of that size, which is how $\alpha$ is defined. Thus $\alpha$ tells us how the length distribution of fragments produced effects the energy density in loops and in gravitational radiation. The effect of the cutoff length for reconnections was studied for $N=10$ by doing runs with several different values of $\ell_{c}$ ranging down to $\ell_{c}=0.1 \ell_{p}$. There was no sign of pathological behavior as $\ell_{c}$ goes to zero. The mean number of fragments produced, $\left\langle N_{f}\right\rangle$, was found to increase from $\left\langle N_{f}\right\rangle=8.6$ without reconnections to $\left\langle N_{f}\right\rangle=17$ for $\ell_{c}=0.1 \ell_{p}$, while $\alpha$ only decreased about $10 \%$. Figure (4) shows $\alpha$ as a function of $N$ both with reconnections and without. The decrease of $\alpha$ with $\mathrm{N}$ is somewhat greater if reconnections are included, but in either case it remains of order unity for the values of $\mathrm{N}$ considered. We see that for these loops the 
important length scale in fragmentation is the persistence length. Whereas in the stochastic fragmentation model the typical fragment length is determined by the parent's length together with the fragmentation probability, for these loops it is essentially set by the persistence length alone.

The time scale for fragmentation is on the order of the period of the parent loop. The mean time of the last intercommutation found was between $0.2 L$ and $0.4 L$ over the range of $N$ and $\ell_{c}$ considered.

The velocities of the fragments affect the evolution of the string in that faster fragments will be more likely to collide with other string, and because the redshifting away of the center of mass motion of fragments removes energy from the string network. Also, structure formation by loops is affected by their motion [1]. Figure (5) shows the mean speed of fragments as a function of $x$. The data plotted is without reconnections; including reconnections left $\langle v\rangle$ the same within the statistical uncertainty. The mean speed of small fragments is about 0.55 , while for larger fragments $\langle v\rangle$ falls of somewhat faster than $x^{-\frac{1}{2}}$, the curve shown in Fig. (5) being $\langle v\rangle=0.7 x^{-0.7}$. The mean fraction of the parent loop's energy which goes into center of mass kinetic energy of the fragments increases from $0.109 \pm .007$ for $N=5$ to $0.170 \pm .006$ for $N=20$, with $\ell_{c}=0.5$; again no statistically significant variation with $\ell_{c}$ was observed.

\section{Conclusions}

For a large class of loops the fragmentation of an isolated loop produces a finite number of non-self-intersecting fragments, in agreement with the findings of Scherrer and Press [18] for a different class of loops. The typical length of these stable fragments is on the order of the persistence length of the parent loop. This behavior is quite different from that predicted by a stochastic model of fragmentation. When the reconnection of fragments which collide is taken into account the typical fragment length decreases somewhat, but the above conclusions still hold. These results strongly suggest that loop fragmentation alone does not cause much energy to flow into loops much shorter than the network's persistence length. This is good news for the numerical simulations of Albrecht and Turok and of Bennett and Bouchet. However it should be emphasized that I have here studied only an isolated parent loop, and the reconnections $I$ have included in the calculation are only those between fragments of that parent loop. Actually, a loop breaking 
off the network can reconnect with long string or with one of the many slowly decaying loops produced at an earlier time. Understanding the reconnection of recently created loops to long string (or to each other) should just require a resolution good compared to the persistence length, $\ell_{p}$, which the numerical simulations have. However, in the scaling solution, most of the energy in string is in loops much shorter than $\ell_{p}$; collisions involving these may have an important effect, but understanding this by simulation would require very high resolution. The understanding of loop fragmentation derived from the simulation described herein should be helpful in constructing a good model of these processes; already it is apparent that reconnections serve to enhance the chopping of string into shorter pieces.

\section{Acknowledgements}

I would like to thank Andy Albrecht for encouragement and numerous helpful discussions, to thank Neil Turok for several stimulating conversations, and to acknowledge the hospitality of Fermilab. This work was supported in part by the DOE and by the NASA at Fermilab.

\section{References}

[1] N. Turok. Phase transitions as the origin of large scale structure of the universe. 1988. Fermilab preprint.

[2] A. Vilenkin. Phys. Rep., 121:265, 1985.

[3] P. Shellard. Nucl. Phys., B283:624, 1987.

[4] R. Matzner. Computers in Physics, 2(5):51, 1988.

[5] N. Turok. Nucl. Phys., B242:520, 1984.

[6] T. Vachaspati and A. Vilenkin. Phys. Rev. D, 31:3052, 1985.

[7] A. Vilenkin. Phys. Rev. D, 23:852, 1981.

[8] D. Garfinkle and T. Vachaspati. Phys. Rev. D, 37:257, 1988.

[9] A. Albrecht and N. Turok. Phys. Rev. Lett., 54:1868, 1985. 


\section{ORIGINAL PAGE IS \\ OF POOR QUALITY}

[10] A. Albrecht and N. Turok. In preparation.

[11] D. Bennett and F. Bouchet. Phys. Rev. Lett., 60:257, 1988.

[12] T.W.B. Kibble. Nucl. Phys., B252:227, 1985.

[13] D. Bennett. Phys. Rev. D, 33:872, 1986.

[14] D. Bennett. Phys. Rev. D, 34:3592, 1986.

[15] E. Copeland and N. Turok. Phys. Lett., B173:129, 1986.

[16] A. L. Chen, D. A. DiCarlo, and S. A. Hotes. Phys. Rev. D, 37:863.

[17] A. Albrecht and T. York. Phys. Rev. D, 38:2958, 1988.

[18] R.J. Scherrer and W.H. Press. Cosmic string loop fragmentation. 1988. Harvard-Smithsonian preprint.

[19] D. Mitchell and N. Turok. Phys. Rev. Lett., 58:1577, 1987.

[20] A.G. Smith and A. Vilenkin. Phys. Rev. D, 36:990, 1987.

[21] T. W. B. Kibble and N. Turok. Phys. Lett., 116B:141, 1982.

[22] A.G. Smith and A. Vilenkin. Phys. Rev. D, 36:987, 1987.

[23] T. York. Unpublished. 


\section{Figure Captions}




\section{ORIGINAL PAGE IS \\ OF POOR QUALITY}

Figure 1: Fragmentation of a typical loop with $N=10$, showing (left) the parent loop shortly before the first self-intersection, and (right) the fragments remaining at end of the fragmentation process. In this case there were 13 self-intersections and 3 reconnections resulting in 11 fragments.

Figure 2: Probability $P_{0}$ that a parent loop has zero self-intersections, as a function of $N$.

Figure 3: The function $\epsilon(x)$, describing the energy going into fragments of different lengths, neglecting reconnections (top), and including them (bottom).

Figure 4: The dependence of $\alpha$ on $N$, with reconnections and without.

Figure 5: The mean speed of fragments as a function of $x$. 


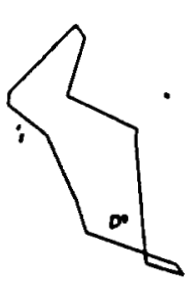

$\Delta$
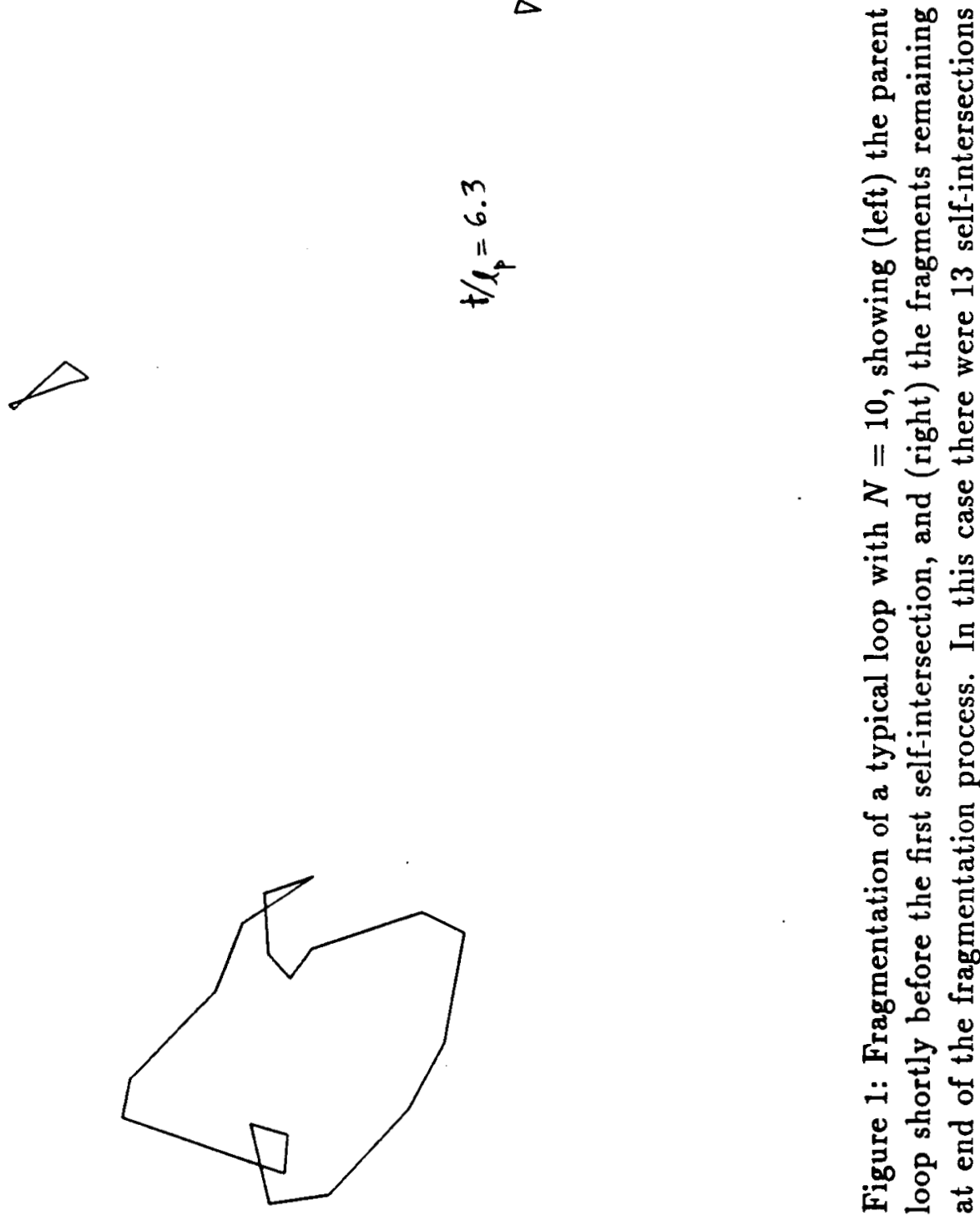

$11 \cdot \frac{0}{2}$

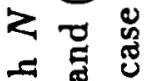

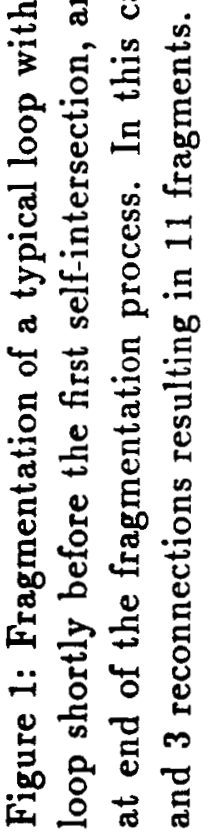

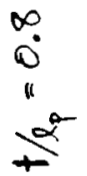


ORIGINAL PAGE IS

OF POOR QUALITY

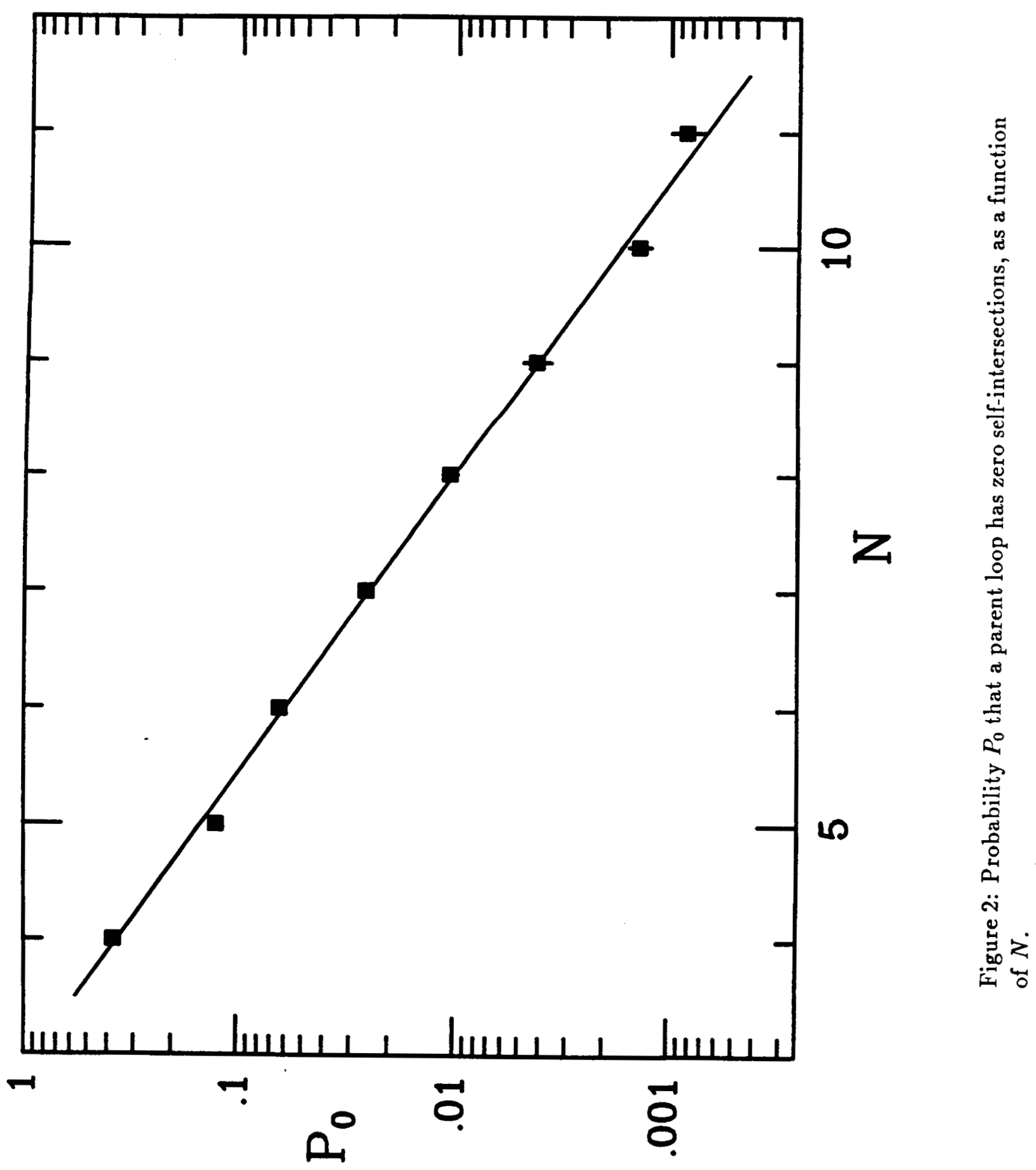




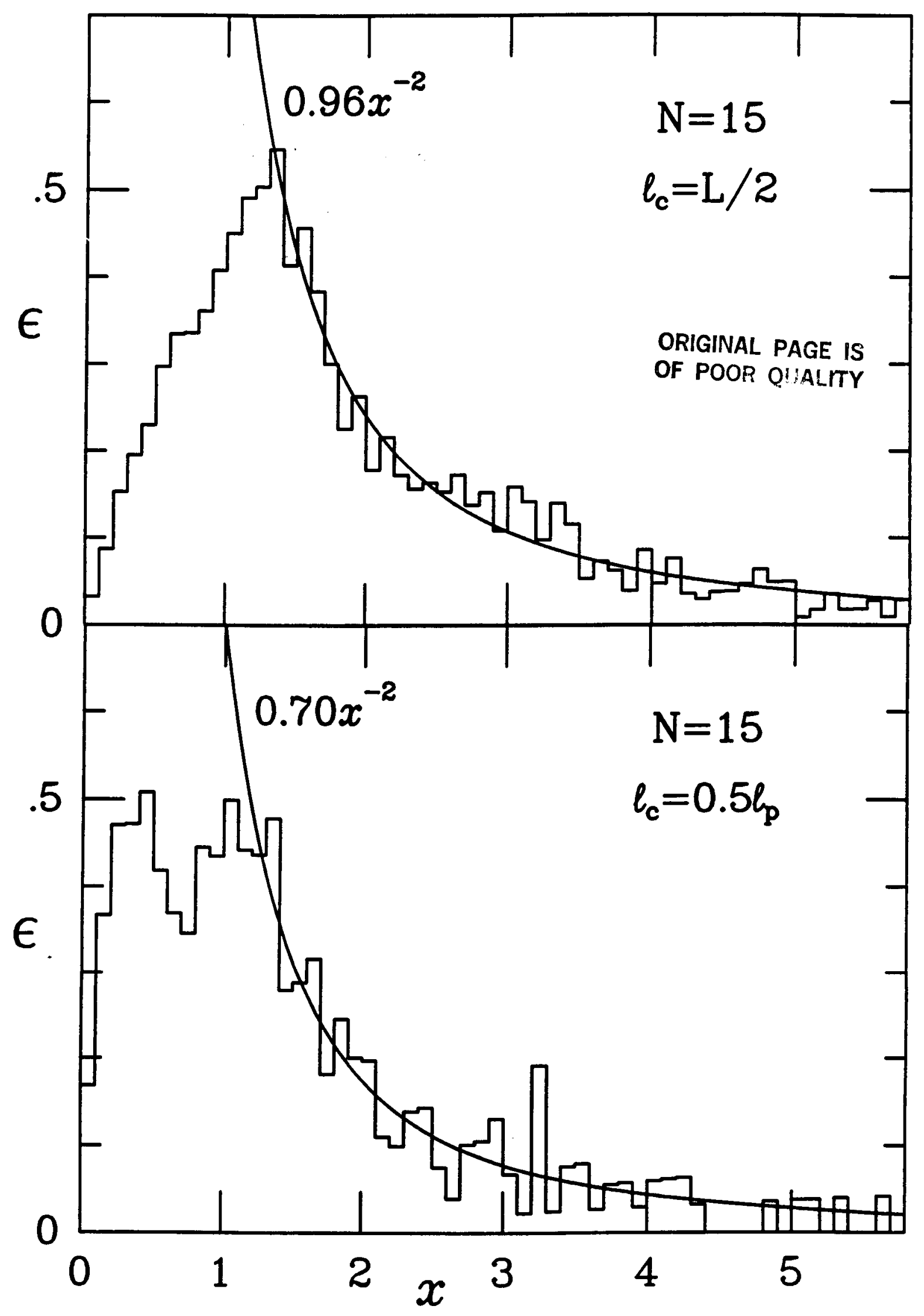

Figure 3: The function $\epsilon(x)$, describing the energy going into fragments of different lengths, neglecting reconnections (top), and including them (bottom). 
ORIGINAL PAGE IS

OF POOR QUALITY

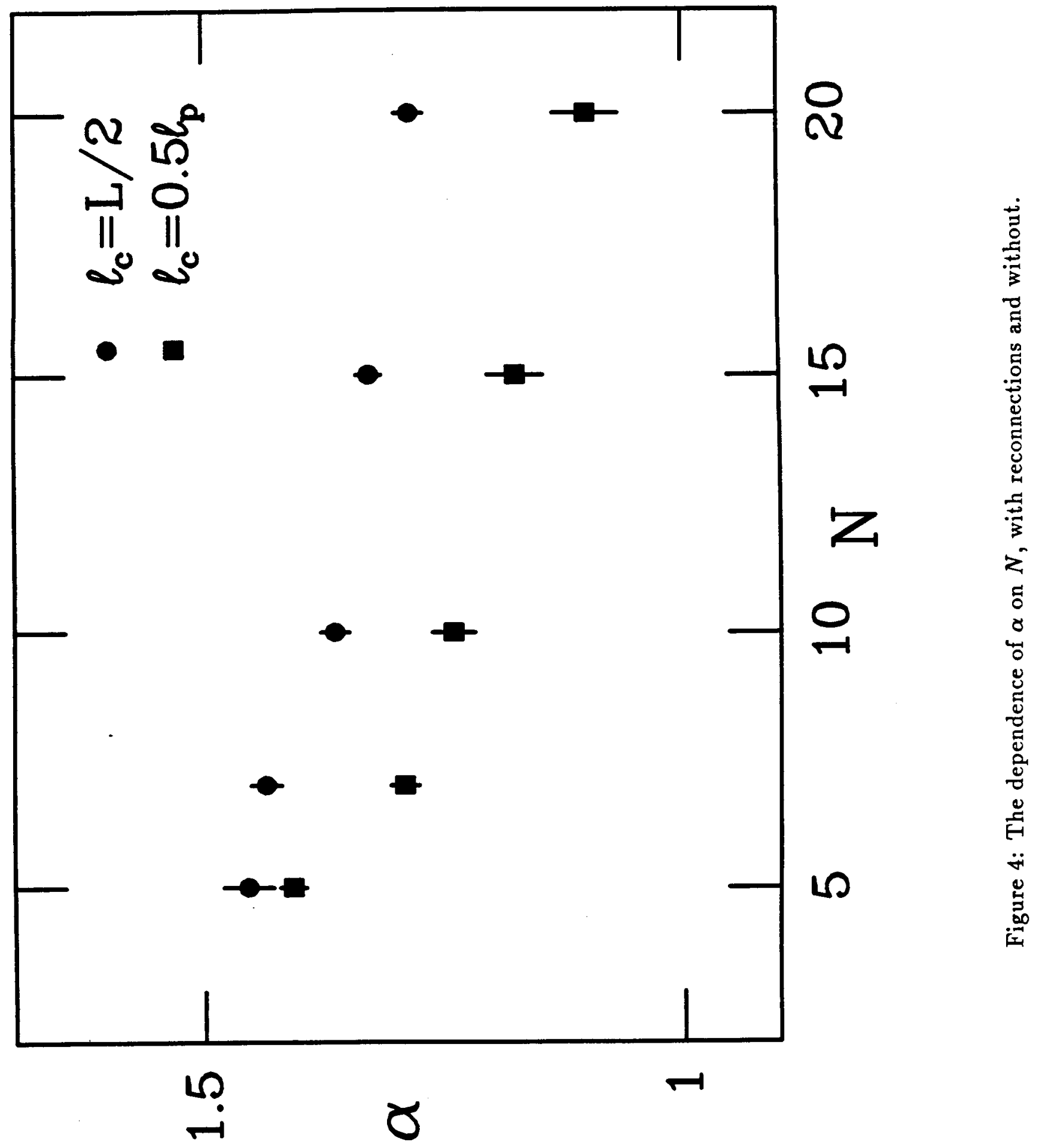




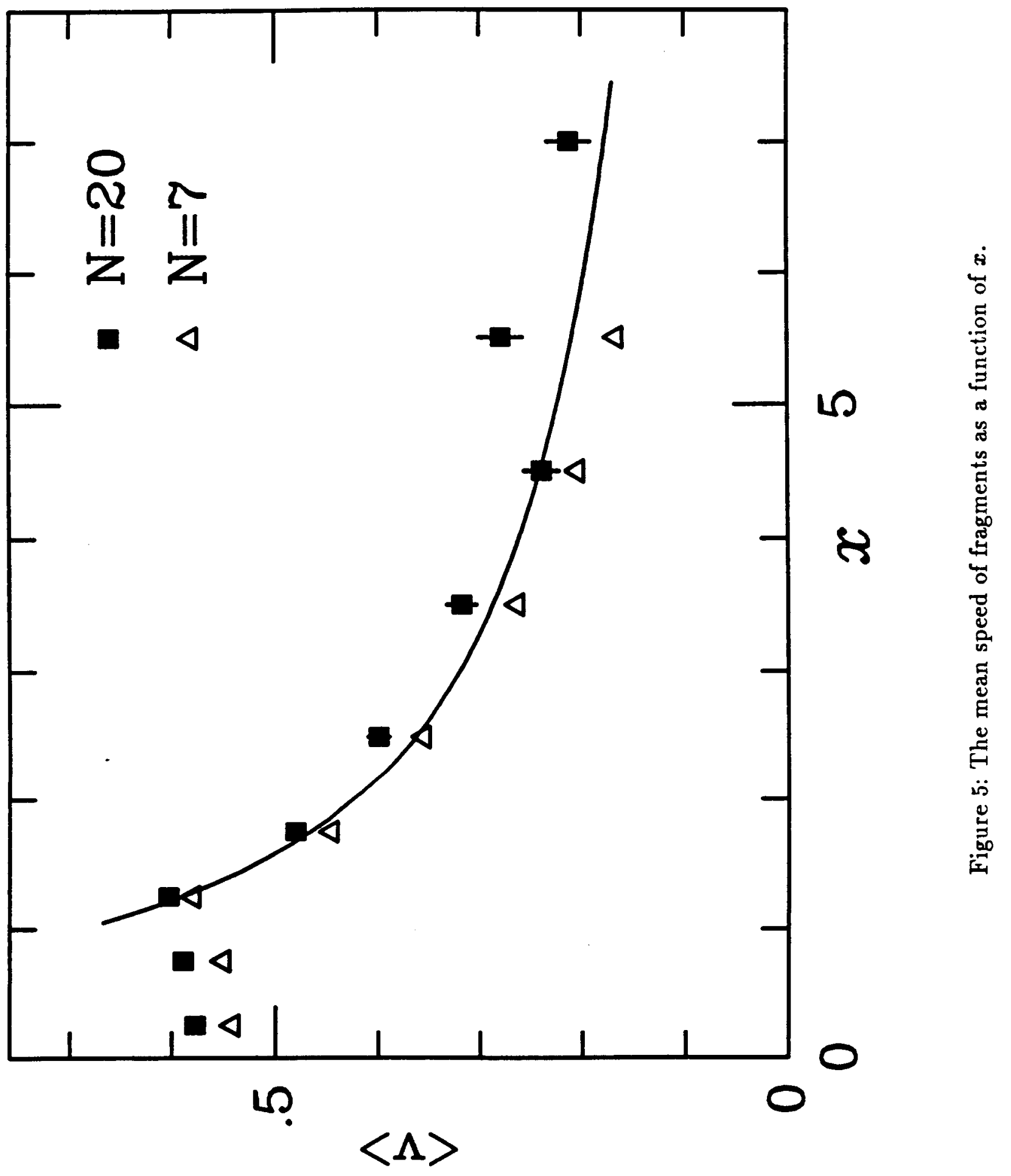

